\title{
Comparative Study of Two Thoracic Radiotherapy Schedules for Palliation of Symptom, Radiological Response and Acute Toxicities in Advanced Non-small Cell Lung Cancer
}

\author{
Subhash Chand Bairwa, Ravinder Singh Gothwal, Badri Ram, Shivani Gupta, \\ Chetna Meena
}

SMS Medical College, Jaipur, India.

\begin{abstract}
Aim: To compare the effect of two RT schedules for thoracic palliation in advanced NSCLC patients (30 Gy in 10 fractions over two weeks and 27 Gy in 6 fractions over three weeks, 2 fractions per week) on pulmonary symptoms, radiological response of the primary thoracic tumour, pulmonary functions and acute toxicities. Material and method: A hospital based quantitative prospective follow-up study. Total 104 advanced NSCLC patients were randomized into two fractionation arms. Evaluation was done pretreatment and 4 weeks after end of RT. Symptoms palliation and radiological response to RT \& radiation pneumonitis were assessed by using RTOG 4-point scale and Revised (RECIST) guideline version 1.1 respectively. Radiotherapy was given by Cobalt-60 teletherapy machine. Results: Total 96 patients were evaluated for symptom palliation, radiological response and acute toxicities. The percentage of patients achieving symptom palliation was slightly higher in the control arm. At $1^{\text {st }}$ month of follow-up, $16.67 \%$ \& $18.75 \%$ patients in Study \& control arm showed PR. Post-RT mean FVC and FEV1 showed a tendency for improvement in both mean FVC and FEV1 in compare to baseline. Treatment was well tolerated both arms. Grade I nausea and vomiting developed in 43\% and 37.5\% patients in Study and Control Arm respectively. 58\% \& 39\% patients developed Grade 1 radiation pneumonitis as cough \& dyspnea in study and control arms respectively. Grade 2 radiation pneumonitis was developed in 2 patient of study arm at $3^{\text {rd }}$ week of follow-up but they lost follow up. Around 31\% patients developed Grade1 esophagitis as dysphagia in both arms at 1 week of follow-up which was reduced up to $7 \%$ by $2^{\text {nd }}$ week. A very few patients developed grade 1 skin reaction as dermatitis in $1^{\text {st }}$ week of treatment. The difference in symptom palliation, radiological response, pulmonary functions and acute toxicities in both arms was not statistically significant. Conclusion: The two RT fractionation schedules showed equal efficacy in terms of symptoms palliation, radiological response of the primary thoracic tumor, pulmonary functions and acute toxicities. Thus the $27 \mathrm{~Gy} / 6$ fractionation arm appears preferable compared to $30 \mathrm{~Gy} / 10$ arm to minimize the patients' visits and load on the machines.
\end{abstract}

Keywords: Non-Small Cell Lung Cancer- Palliative Thoracic Radiation- Hypofractionation Schedules- Symptoms

\section{Introduction}

Worldwide, lung cancer is the most common and the deadliest form of cancer accounts for $11.6 \%$ (2.09 million) of the total cases of cancer and $18.4 \%$ (1.76 million) of the cancer-related deaths based on 2018 GLOBOCAN estimates [1]. Among males, lung cancer is the most commonly diagnosed cancer (14.5\%)and leading cause of cancer death $(22.0 \%)$. In India, currently lung cancer is the fourth largest cause of cancer accounting for nearly $6.45 \%$ of all cancer related deaths in the country [2]. Among males, it is the leading cause of cancer mortality, accounting for $11 \%$ of all cancer deaths. Histologically, lung cancer can be divided into small cell lung cancer 
(SCLC) and non-small cell lung cancer (NSCLC) accounting for $15 \%$ and $85 \%$ respectively. Among NSCLC, most common histologies are adenocarcinoma (50-60\% cases), squamous cell carcinoma (20-25\%).

The main goals of treatment in advanced NSCLC patients are prolongation of life, palliation of symptoms such as pain, dyspnea, cough, hemoptysis, chest pain, airway obstruction or SVC obstruction syndrome [3]. In any stage of NSCLC, smoking cessation should be highly encouraged because it improves the outcome [4]. Radiotherapy is often used as a palliative treatment for patients with stage IV NSCLC to relieve symptoms (i.e. hemoptysis, cough, chest pain, dyspnea, etc.) that are caused by loco-regional growth of primary tumor [5]. For intrathoracic disease with an obstructive component, 30 to 45 Gy in 2.5- to 3-Gy fractions over 2 to 3 weeks is generally recommended [6]. For patients with poor performance status or for whom daily radiotherapy over 2 to 3 weeks is logistically difficult, hypofractionated regimens (of 1 to 5 fractions) have been utilized with good palliative results [7-8].

This study was intended to compare two different palliative thoracic radiotherapy fractionation schedules in advanced stage non small cell lung cancer patients in favour of symptom control, radiological response and pulmonary function profile

\section{Materials and Methods}

It was a hospital based quantitative prospective follow-up study which was conducted in year 2018-2019 in a tertiary care center of north- west India. After approval of institutional Review Board/ Ethical committee, histopathological proven advanced non-small cell lung cancer (stage IVA\&IVB) patients who required palliative radiotherapy and ready to give informed written consent were included in this study. Sample size was 48 cases in each group are required a sample size which is enhanced to 53 expecting $10 \%$ drop outs, calculated at $80 \%$ study power at $\alpha$ error of 0.05 . Patients with previous history of thoracic irradiation or with history any co-morbidity were excluded from study population.

\section{Randomization}

Eligible patients were randomized into two fractionation arms: 30 Gy in 10 fractions over two weeks and 27 Gy in 6 fractions over three weeks (two fractions per week).

\section{Evaluation}

Evaluation was done pretreatment and 4 weeks after end of RT in the form of: Complete history, physical examination. Symptomatic assessment using a Radiotherapy Oncology Group (RTOG) 4-point scale (none, mild, moderate, severe/Gr 0-3). Palliation of a symptom was defined as disappearance or improvement of the initial symptom one or more degree along the scale. Radiological response to RT (longest diameter was recorded) and radiation pneumonitis assessment was done according to: New Response Evaluation Criteria In Solid Tumors: Revised (RECIST) guideline version 1.1 [9].
Acute toxicity (esophagitis and skin reaction) assessment was done according to Radiotherapy Oncology Group (RTOG) scale. A baseline and 4 weeks post RT Pulmonary function test was done. On subsequent follow up at $3^{\text {rd }}$ month detailed systemic examinations, CBC, LFT, RFT, chest $\mathrm{X}$-ray was done to evaluate for acute toxicities.

Primary end points encompassing palliation of chest tumor related symptoms, pulmonary functions: Forced vital capacity (FVC), Forced Expiratory Volume1 (FEV1) and radiological response of the primary thoracic tumor. Whereas the secondary end point aimed at comparing the treatment side effects relative to each fractionation arm.

\section{RT Technique}

The patients in both arms were simulated and treated in supine position with arms up and antero-posterior parallel opposed pair of iso-centric fields that encompassed the primary tumor and involved lymph nodes with a margin of $2 \mathrm{~cm}$. Maximum possible uninvolved area of normal lung tissue was shielded. The dose was prescribed to the mid-plane in the centre of the fields. External beam radiotherapy was given by Cobalt-60 teletherapy machine. Treatment interruptions were strongly discouraged. Treatment breaks were noted in the treatment record and appropriate time correction was made.

In the study arm, patients received a total of $27 \mathrm{~Gy}$ in 6 fractions over three weeks, two fractions per week (with 4.5 Gy per fraction) and in the control arm, patients received a total of 30 Gy in 10 fractions over two weeks (with 3 Gy per fraction).

\section{Statistical analysis}

Quantitative data were expressed in means with standard deviation and qualitative data were expressed in percentage proportions. Significance of difference in means of two groups was inferred with unpaired T test. Signification of difference in means at various follow up period was inferred with repeated ANOVA test. Significance of difference in proportion in two groups was inferred with Chi-square test. For significance P value less than 0.05 were considered as significance.

\section{Results}

\section{Observations}

This study included 96 Patients with advanced NSCLC who presented to the Department of radiotherapy at S.M.S. Medical College and Attached Groups of Hospitals, Jaipur from June 2018 to August 2019 for palliative irradiation to the lung excluding drop outs and evaluated for symptom palliation, radiological response, pulmonary function and acute toxicities profile.

\section{Patient Criteria}

Both arms were well balanced regarding age, sex, smoking habit, co morbidity, ECOG- PS HB level, PS, histopathology and stage group (Table 1). Squamous cell carcinoma was most common histology 52 [54.2\%] followed by Adenocarcinoma 37 [38.5\%]. Other NSCLC 


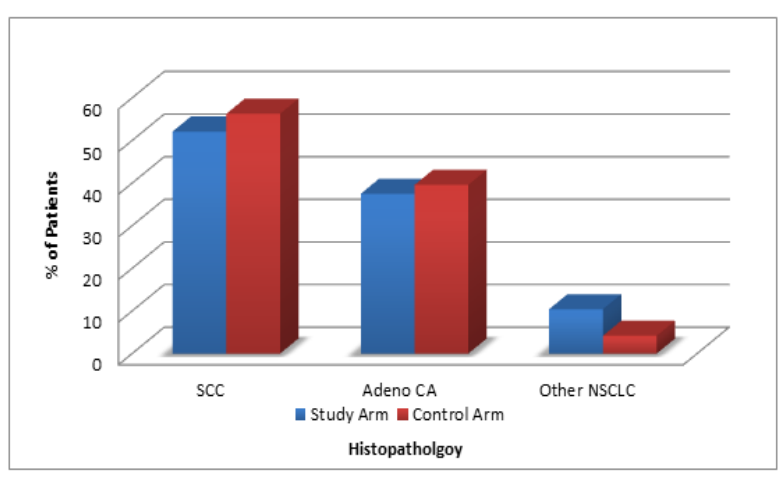

Figure 1. Comparison between the two Studied Groups According to Histopathology

histopathology variety accounted 7 [7.3\%] (Figure 1).

\section{Baseline symptoms}

Chest pain was the most common symptom of presentation 80 (83\%) followed by cough 74 (77\%), dyspnea $74(77 \%)$ and hemoptysis $31(33 \%)$. There was no significant difference between both arms in term of the incidence or the degree of thoracic symptoms (Table 2).

\section{Response Evaluation}

Symptom palliation

The percentage of patients achieving improvement

Table 1. Patient Criteria

\begin{tabular}{|c|c|c|c|}
\hline & $\begin{array}{c}\text { Study Arm } \\
\text { N (\%) }\end{array}$ & $\begin{array}{c}\text { Control Arm } \\
\mathrm{N}(\%)\end{array}$ & $P$ value \\
\hline \multicolumn{4}{|l|}{ Criteria } \\
\hline \multicolumn{4}{|l|}{ AGE } \\
\hline Range & $41-78$ & $43-76$ & \\
\hline Mean & 59.16 & 58.97 & 0.683 \\
\hline \multicolumn{4}{|l|}{ SEX } \\
\hline Male & $45(93.7 \%)$ & $44(91.7 \%)$ & \\
\hline Female & $3(6.3 \%)$ & $4(8.3 \%)$ & 0.694 \\
\hline Smoking & $44(91.7 \%)$ & $42(87.5 \%)$ & 0.763 \\
\hline $\mathrm{DM}$ & $3(6.3 \%)$ & $2(4.1 \%)$ & 0.645 \\
\hline HTN & $4(8.3 \%)$ & $6(12.5 \%)$ & 0.501 \\
\hline \multicolumn{4}{|l|}{$\mathrm{Hb}$} \\
\hline Range & $9.1-31.7 \mathrm{gm} \%$ & $9.3-14.0 \mathrm{gm} \%$ & 0.765 \\
\hline Mean & $12.3 \mathrm{gm} \%$ & $12.5 \mathrm{gm} \%$ & \\
\hline \multicolumn{4}{|l|}{ ECOG-PS } \\
\hline 1 & $28(58.4 \%)$ & $7(14.6 \%)$ & 0.473 \\
\hline 2 & $10(20.8 \%)$ & $30(62.5 \%)$ & \\
\hline 3 & $10(20.8 \%)$ & $11(22.9 \%)$ & \\
\hline \multicolumn{4}{|c|}{ Histopathology } \\
\hline $\mathrm{SqCC}$ & $25(52.1 \%)$ & $27(56.2 \%)$ & 0.499 \\
\hline AdenoCa & $18(37.5 \%)$ & $19(39.6 \%)$ & \\
\hline \multicolumn{4}{|l|}{ Stage Group } \\
\hline IVA & $13(27.1 \%)$ & $12(2 \%)$ & 0.473 \\
\hline IVB & $35(72.9 \%)$ & $36(7 \%)$ & \\
\hline
\end{tabular}

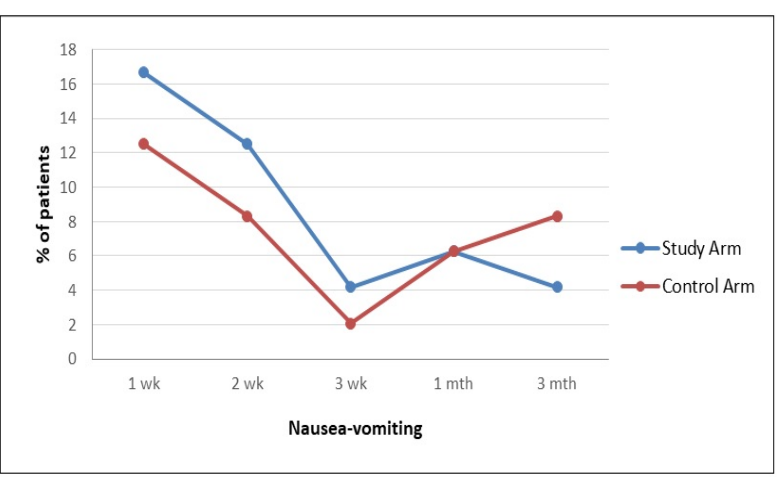

Figure 2. Post RT Acute Toxicity (Nausea/Vomiting) Comparison between two Studied Groups

in symptoms (namely cough chest pain, dyspnea or hemoptysis) was slightly higher in the control arm, but did not reach statistical significance for any symptom (Table 3).

\section{Radiological Response (PR/SP/PD)}

The mean longest tumor diameter recorded in pre treatment CT was $7.12 \mathrm{~cm}$ in Control Arm compared to $7.45 \mathrm{~cm}$ in Study Arm without significant difference $(\mathrm{P}$ value $=0.68)$. At $1^{\text {st }}$ month follow-up, $83.33 \%$ patients in study arm \& $81.25 \%$ patients in control arm had progressive/stable disease while $16.67 \%$ patients in study arm \& $18.75 \%$ patients in control arm had regression of disease but difference in both arms was not statistically significant (Table 4).

\section{Pulmonary function test}

At base line, patients in both arms had comparable mean FVC and FEV1. Post 1 month of RT mean FVC and FEV1 showed a tendency for improvement in both mean FVC and FEV1 in compare to baseline. However this improvement didn't reach statistical significance in each arm (Table 5).

\section{Acute toxicity}

Treatment was generally well tolerated in both arms. Grade I nausea and vomiting developed in $43 \%$ and $37.5 \%$ patients in Study and Control Arm respectively. No grade II/III/IV anorexia, nausea and vomiting were

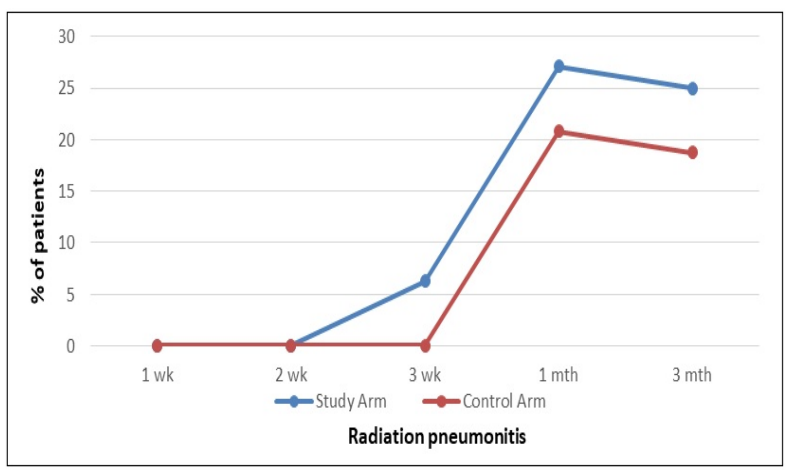

Figure 3. Post RT Pneumonitis Comparison between two Studied Groups 
Table 2. Baseline Symptom

\begin{tabular}{|c|c|c|c|c|}
\hline Symptoms & Grade & $\begin{array}{c}\text { Study arm } \\
\text { N (\%) }\end{array}$ & $\begin{array}{c}\text { Control Arm } \\
\mathrm{N}(\%)\end{array}$ & $\mathrm{p}$ value \\
\hline \multirow[t]{4}{*}{ Chest Pain } & Grade 0 & $6(12.5 \%)$ & $10(20.8 \%)$ & 0.314 \\
\hline & Grade 1 & $7(14.6 \%)$ & $10(20.8 \%)$ & $\chi^{2}=3.551$ \\
\hline & Grade 2 & $23(47.9 \%)$ & $22(45.8 \%)$ & \\
\hline & Grade 3 & $12(2 \%)$ & $6(12.5 \%)$ & \\
\hline \multirow[t]{4}{*}{ Cough } & Grade0 & $9(18.7 \%)$ & $13(27.1 \%)$ & 0.227 \\
\hline & Grade 1 & $15(31.2 \%)$ & $7(14.6 \%)$ & $\chi 2=4.37$ \\
\hline & Grade 2 & $18(37.5 \%)$ & $23(47.9 \%)$ & \\
\hline & Grade 3 & $6(12.5 \%)$ & $5(10.4 \%)$ & \\
\hline \multirow[t]{4}{*}{ Dyspnea } & Grade0 & $13(27.1 \%)$ & $9(18.7 \%)$ & 0.79 \\
\hline & Grade 1 & $9(18.7 \%)$ & $10(20.8 \%)$ & $\chi 2=1.023$ \\
\hline & Grade 2 & $17(35.4 \%)$ & $20(41.7 \%)$ & \\
\hline & Grade 3 & $9(18.7 \%)$ & $9(18.7 \%)$ & \\
\hline \multirow[t]{4}{*}{ Hemoptysis } & Grade0 & $32(66.7 \%)$ & $33(68.7 \%)$ & 0.85 \\
\hline & Grade 1 & $11(22.9 \%)$ & $9(18.7 \%)$ & $\chi 2=0.306$ \\
\hline & Grade 2 & $5(10.4 \%)$ & $6(12.5 \%)$ & \\
\hline & Grade 3 & 0 & 0 & \\
\hline
\end{tabular}

(None, mild, moderate, severe/Gr 0-3)

noticed in either of the arms (Figure 2). Around 31\% patients developed Grade1 esophagitis as dysphagia in both arms at 1 week of follow-up which was reduced up to $7 \%$ by $2^{\text {nd }}$ week. Most of patients with dysphagia, were associated with hilar or mediastinal disease. A Very few patients developed grade1 skin reaction as dermatitis in $1^{\text {st }}$ week of treatment.

$58 \%$ \& 39\% patients developed Grade 1 radiation pneumonitis as cough \& dyspnea in study and control arms respectively. Grade 2 radiation pneumonitis was developed in 2 patient of study arm at $3^{\text {rd }}$ week of follow-up but they lost follow up. The radiological finding was a diffuse haziness or fuzziness in areas of

Table 3. Symptom Palliation

\begin{tabular}{lccc}
\hline & Study Arm N & Control Arm N & p value \\
\hline Chest Pain & & & \\
Improvement & $34 / 42$ & $33 / 38$ & 0.772 \\
Progression & $3 / 42$ & $2 / 38$ & $\chi 2=0.516$ \\
SD & $5 / 42$ & $3 / 38$ & \\
Cough & & & \\
Improvement & $31 / 39$ & $29 / 35$ & 0.771 \\
Progression & $4 / 39$ & $2 / 35$ & $\chi 2=0.518$ \\
SD & $4 / 39$ & $4 / 35$ & \\
Dyspnea & & & \\
Improvement & $29 / 35$ & $33 / 39$ & 0.783 \\
Progression & $1 / 35$ & $2 / 39$ & $\chi 2=0.487$ \\
SD & $5 / 35$ & $4 / 39$ & \\
Hemoptysis & & & \\
Improvement & $14 / 16$ & $13 / 15$ & 0.997 \\
Progression & $1 / 16$ & $1 / 15$ & $\chi 2=0.0048$ \\
SD & $1 / 16$ & $1 / 15$ & \\
\hline
\end{tabular}

the irradiated lung. The difference in all acute toxicities in both arms was not statistically significant (Figure 3).

\section{Discussion}

The issue of optimal palliative irradiation schedule in advanced symptomatic NSCLC has been a subject of numerous randomized studies. There is a debate about the optimal fractionation scheme to be used; some randomized studies favor a hypo fractionation treatment policy [10]. Others do not recommend hypo fractionation because of the increased toxicity and/or reduced survival [9-11]. In our study the fractionation in Control Arm 27 Gy/6 over 3 weeks was used only in very few previous randomize studies and the long overall treatment time was intended to minimize toxicity of short fractionation schedules and it proved same palliation.

Keller et al, [12] examined the influence of gender in the ECOG trial and did not find any significant difference in survival or compliance. In our study, we restricted ECOG PS up to 3. In many similar studies patients of any age or PS were included however Simpson et al [13] excluded patients above 75 years. Although age has not been shown to be an independent prognostic factor, it may reflect co-morbidity and give information about

Table 4. Radiological Response (PR/SP/PD)

\begin{tabular}{lcc}
\hline & \multicolumn{2}{c}{ No. of Patients (\%) } \\
& Study Arm N (\%) & Control Arm N (\%) \\
\hline PD & $17(35.4 \%)$ & $19(39.6 \%)$ \\
PR & $8(16.7 \%)$ & $9(18.7 \%)$ \\
SD & $23(47.9 \%)$ & $20(41.7 \%)$ \\
p value & $0.827, \chi 2=0.379$ & \\
\hline
\end{tabular}


Table 5. Pre and Post RT Pulmonary Function Test

\begin{tabular}{lccccc}
\hline & \multicolumn{2}{c}{ Study Arm: Mean(SD) } & \multicolumn{2}{c}{ Control Arm :Mean(SD) } & p value \\
\hline & PFT BASELINE & PFT at 1 month post RT & PFT BASELINE & PFT at 1 month post RT \\
FVC & $53.96(11.27)$ & $58.04(10.46)$ & $53.75(11.35)$ & $58.19(10.66)$ & 0.928 \\
FEV1 & $50.35(10.64)$ & $53.90(9.70)$ & $50.42(10.63)$ & $54.02(9.96)$ & 0.977 \\
\hline
\end{tabular}

case selection.

Review of literature on symptomatology highlights diverse symptomatic presentation of lung cancer. Cough, chest pain, weight loss, dyspnea, hemoptysis are most common presentations and rare ones are stridor, change in voice, dysphagia, fatigue, anorexia, pain in other body parts [14]. Our study population is consistent with reported data. Chest pain (83\%), Dyspnea (77\%) Cough (77\%) and hemoptysis $(33 \%)$ were most common presentation; dysphagia, fever, neck swelling, pain in other parts were uncommon presentation (5\%). Palliation of symptoms was slightly better and was for longer duration in control arm, though early palliation was achieved in study arm. It was observed that in patients with good performance status palliation achieved was better. This was similar to other authors $[10,13,15]$ who found that the most effectively palliated symptoms were chest pain and haemoptysis.

Sundstrom $\mathrm{S}$ et al. [15] evaluated three treatment arms (17Gy/2Fr; 42Gy/15Fr; and 50Gy/25Fr), concluded that protracted palliative TRT renders no improvement in symptom relief, HRQOL, or survival when compared with short-term hypo-fractionated treatment in NSCLC Patients with disease too advanced for curative RT (stage III \& IV patients were included). The effects of hypo-fractionated thoracic radiotherapy (TRT) were comparable to more standard fractionated RT. Symptom relief and HRQOL were equivalent in all the treatment arms. In contrast of our study, there was no limitation of age and PS was 0 - 3 . Clinicians' assessments of symptom improvement were at 2, 6, and 14 weeks after completion of treatment. Also Macbeth et al. [16] reported that no strong evidence for 1223 weqweqthe superiority of any particular regimen in spite of differences in the radiotherapy regimens, patient characteristics and outcome measures. Senkus-Konefka, et al. [17] randomized 100 patients into 2 arms: 20 Gy in 5 daily fractions (arm A: 55 patients) or 16 Gy in 2 fractions, day 1 and 8 (arm B: 45 patients). The grading of symptom intensity was performed using a 4-point scale (none, mild, moderate, severe). No significant differences between study arms were observed. This idea of non superiority of any particular regimen in thoracic palliation of advanced lung cancer was confirmed by a Cochrane analysis of 10 randomized palliative radiotherapy trials indicated that symptomatic relief was equivalent regardless of the total radiotherapy dose [16]. Van den Hout et al. [18] compared two fractionation arms (30 Gy in 10 fractions in 2 weeks and 17 Gy in 2 fractions 1week apart) and reported that arms were equally effective regarding palliation. This is similar to Rees et al. [10] who also found that $17 \mathrm{~Gy}$ in 2 fractions, day 1 and 8 or 22.5 Gy in five daily fractions had no clinically important differences in efficacy between the two regimens. Erridge et al. [19] reported that the 30 Gy in 10 fractions over 2 weeks regimen was significantly better at reducing chest pain and dyspnea compared to 10 Gy single fraction regimens. In addition, a significant improvement in PS with fractionated regimen.

In our study, $16.67 \%$ patients in study arm \& $18.75 \%$ patients in control arm had PR of the primary thoracic lesion 4 weeks after treatment on evaluation by $\mathrm{CT}$ chest This was similar to MRC. [20] in which $22 \%$ of arm A (17 Gy in 2 fractions, day 1 and 8 ) and $25 \%$ of arm B (30 Gy in 10 fractions in 2 weeks or 27 Gy in 6 fractions in 8 days) achieved PR without statistical significant. Senkus-Konefka et al. [17] also reported equality between two compared arms (20 Gy in 5 fractions in 1 week and 16 Gy in 2 fractions, day 1 and 8) regarding radiological assessment of primary thoracic lesion by chest X ray 2 weeks after treatment. In this study, 52\% and $54 \%$ achieved PR in the 5 fractions regimen and the single fraction regimen respectively without significant difference.

In this study $43 \%$ and $37.5 \%$ patient in Study and Control Arm developed Grade I nausea and vomiting respectively. Around 31\% patients developed Grade1 esophagitis as dysphagia in both arms at 1 week of follow-up which was reduced up to $7 \%$ by 2 nd week. Most of patients with dysphagia, were associated with hilar or mediastinal disease. Grade I skin toxicity was seen in $2 \& 4$ patients in Study \& Control arms only at $1^{\text {st }}$ week of treatment. This is similar to Bezjak et al. [21] who reported mild esophagitis and skin reaction without significant difference between the two treatment arms. This is similar to Lupatelli et al. [22] Study in which only 4 patients (5\%) experienced World Health Organization grade III dysphagia. No reported cases of skin reaction. This is similar to Cross et al. [23] who reported no cases of radiation esophagitis or skin reaction in $16 \mathrm{~Gy}$ in 2 fractions, day 1 and 8 regimen. This is in contrast to Rees et al.[10] who found that $50 \%$ of patients receiving two fractions (17 Gy over 2 fractions, day 1 and 8 ) and 38\% of those having five fractions (22.5 Gy in 5). At 3 weeks after treatment, only $7 \%$ of all patients reported moderate/ severe dysphagia required enteral and parenteral support.

In our study $58 \%$ \& $39 \%$ patients developed Grade 1 radiation pneumonitis as cough \& dyspnea in study and control arms respectively with no significant difference. Grade 2 radiation pneumonitis was developed in 2 patient of study arm at $3^{\text {rd }}$ week of follow-up but they lost follow up. This is in contrast to Nestle et al. [11] study in which 152 patients were randomized to receive conventionally fractionated (arm A: 60 Gy in 30 fractions over 6 weeks, number of patients: 79) or short term accelerated treatment (arm B: 32 Gy, 2 Gy bid in 8 days; number of patients: 73). $65 \%$ of patients had clear or equivocal radiological signs of pulmonary radiation injury 6 weeks after treatment. Such a high incidence in this 
study can be easily linked to much higher RT doses used.

In conclusion, our study demonstrated the equal efficacy of the two palliative lung cancer radiotherapy schedules (30 Gy/10 fractions/2 weeks regimen and 27 Gy/6 fractions/3 weeks, 2 fractions per week regimen) in terms of palliative effect, radiological response of the primary thoracic tumor, respiratory functions and toxicity. It involves weekly two attendances for three week would to be of great value in the palliative treatment of remote patients.

Therefore, for advanced NSCLC patients with poor PS and short life-span, the palliative hypo-fractionated regimen of short duration $(27 \mathrm{~Gy} / 6 \mathrm{fr})$ could be considered as a reasonable alternative for a substantial palliation and lesser treatment related morbidity or may be suitable alternative in our busy department and is therefore much more cost-effective in terms of machine-time and staff. Thus we intend to implement this regimen on a bigger number of study group patients with longer follow up to validate it to become a suitable alternative in our busy department.

\section{Limitations of the Study}

Small number of patients in each randomization arms and short follow up.

\section{References}

1. Global cancer statistics 2018: GLOBOCAN estimates of incidence and mortality worldwide for 36 cancers in 185 countries.

2. cancerindia.org.in/globocan-2018-india-factsheet/.

3. Zarogoulidis K, Zarogoulidis P, Darwiche K, et al. Treatment of Non-Small Cell Lung Cancer (NSCLC). Journal of Thoracic Disease. 2013;5:S389-S396.

4. Peters S, Adjei A, Gridelli C, Reck M, Kerr K, Felip E. Metastatic non-small-cell lung cancer (NSCLC): ESMO Clinical Practice Guidelines for diagnosis, treatment and follow-up. Annals of Oncology. 2012 Oct;23:vii56-vii64. https://doi.org/10.1093/annonc/mds226

5. Kramer GW, Wanders SL, Noordijk EM, Vonk EJ, van Houwelingen HC, van den Hout WB, Geskus RB, Scholten M, Leer JH. Results of the Dutch National Study of the Palliative Effect of Irradiation Using Two Different Treatment Schemes for Non-Small-Cell Lung Cancer. Journal of Clinical Oncology. 2005 05 01;23(13):2962-2970. https://doi.org/10.1200/jco.2005.01.685

6. Fairchild A, Harris K, Barnes E, Wong R, Lutz S, Bezjak A, Cheung P, Chow E. Palliative Thoracic Radiotherapy for Lung Cancer: A Systematic Review. Journal of Clinical Oncology. 200808 20;26(24):4001-4011. https://doi. org/10.1200/jco.2007.15.3312

7. Reinfuss M, Mucha-Małecka A, Walasek T, Blecharz P, Jakubowicz J, Skotnicki P, Kowalska T. Palliative thoracic radiotherapy in non-small cell lung cancer. An analysis of 1250 patients. Palliation of symptoms, tolerance and toxicity. Lung Cancer. 2011 03;71(3):344-349. https://doi. org/10.1016/j.lungcan.2010.06.019

8. Metcalfe SK, Milano MT, Bylund K, Smudzin T, Rubin P, Chen Y. Split-Course Palliative Radiotherapy for Advanced Non-small Cell Lung Cancer. Journal of Thoracic Oncology. 2010 02;5(2):185-190. https://doi.org/10.1097/ jto.0b013e3181c6eb20
9. Eisenhauer E, Therasse P, Bogaerts J, Schwartz L, Sargent D, Ford R, Dancey J, Arbuck S, Gwyther S, Mooney M, Rubinstein L, Shankar L, Dodd L, Kaplan R, Lacombe D, Verweij J. New response evaluation criteria in solid tumours: Revised RECIST guideline (version 1.1). European Journal of Cancer. 2009 01;45(2):228-247. https://doi.org/10.1016/j. ejca.2008.10.026

10. Rees G, Devrell C, Barley V, Newman H. Palliative radiotherapy for lung cancer: Two versus five fractions. Clinical Oncology. 1997 01;9(2):90-95. https://doi. org/10.1016/s0936-6555(05)80446-7

11. Nestle U, Nieder C, Walter K, Abel U, Ukena D, Sybrecht $\mathrm{GW}$, Schnabel K. A palliative accelerated irradiation regimen for advanced non-small-cell lung cancer VS. conventionally fractionated $60 \mathrm{GY}$ : results of a randomized equivalence study. International Journal of Radiation Oncology*Biology*Physics. 2000 08;48(1):95-103. https:// doi.org/10.1016/s0360-3016(00)00607-6

12. Keller S, Vangel M, Adak S, Wagner H, Schiller J, Herskovic A, Komaki R, Perry M, Marks R, Livingston R, Johnson D. The influence of gender on survival and tumor recurrence following adjuvant therapy of completely resected stages II and IIIa non-small cell lung cancer. Lung Cancer. 2002 09;37(3):303-309. https://doi.org/10.1016/s01695002(02)00103-4

13. Simpson J, Francis M, Perez-Tamayo R, Marks R, Rao D. Palliative radiotherapy for inoperable carcinoma of the lung: Final report of a rtog multi-institutional trial. International Journal of Radiation Oncology*Biology*Physics. 1985 04;11(4):751-758. https://doi.org/10.1016/03603016(85)90307-4

14. Spiro SG, Gould MK, Colice GL. Initial Evaluation of the Patient With Lung Cancer: Symptoms, Signs, Laboratory Tests, and Paraneoplastic Syndromes. Chest. 2007 09;132(3):149S-160S. https://doi.org/10.1378/chest.07-1358

15. Sundstrøm S, Bremnes R, Aasebø U, Aamdal S, Hatlevoll R, Brunsvig P, Johannessen DC, Klepp O, Fayers PM, Kaasa S. Hypofractionated Palliative Radiotherapy (17 Gy per two fractions) in Advanced Non-Small-Cell Lung Carcinoma Is Comparable to Standard Fractionation for Symptom Control and Survival: A National Phase III Trial. Journal of Clinical Oncology. 200403 01;22(5):801-810. https://doi. org/10.1200/jco.2004.06.123

16. Lester J, Macbeth F, Toy E, Coles B. Palliative Radiotherapy Regimens for Non- Small Cell Lung Cancer. Cochrane Database of Systematic Reviews, CD002143. 2004.

17. Senkus-Konefka E, Dziadziuszko R, Bednaruk-Młyński E, Pliszka A, Kubrak J, Lewandowska A, Małachowski K, Wierzchowski M, Matecka-Nowak M, Jassem J. A prospective, randomised study to compare two palliative radiotherapy schedules for non-small-cell lung cancer (NSCLC). British Journal of Cancer. 2005 03;92(6):10381045. https://doi.org/10.1038/sj.bjc.6602477

18. van den Hout WB, Kramer GWPM, Noordijk EM, Leer JH. Cost-Utility Analysis of Short- Versus Long-Course Palliative Radiotherapy in Patients With Non-Small-Cell Lung Cancer. JNCI: Journal of the National Cancer Institute. 2006 Dec 20;98(24):1786-1794. https://doi.org/10.1093/ jnci/djj496

19. Erridge S, Gaze M, Price A, Kelly C, Kerr G, Cull A, MacDougall R, Howard G, Cowie V, Gregor A. Symptom control and quality of life in people with lung cancer: a randomised trial of two palliative radiotherapy fractionation schedules. Clinical Oncology. 2005 02;17(1):61-67. https:// doi.org/10.1016/j.clon.2004.09.008

20. Medical Research Council Lung Cancer Working Party 
(1991) Inoperable Non-Small-Cell Lung Cancer (NSCLC): A Medical Research Council Randomized Trial of Palliative Radiotherapy with Two Fractions or Ten Fractions. British Journal of Cancer.63:265-70.

21. Bezjak A, Dixon P, Brundage M, Tu DS, Palmer MJ, Blood P, Grafton C, Lochrin C, Leong C, Mulroy L, Smith C, Wright J, Pater JL. Randomized phase III trial of single versus fractionated thoracic radiation in the palliation of patients with lung cancer (NCIC CTG SC.15). International Journal of Radiation Oncology*Biology*Physics. 2002 Nov;54(3):719-728. https://doi.org/10.1016/s03603016(02)02989-9

22. Lupattelli M, Maranzano E, Bellavita R, Chionne F, Darwish S, Piro F, Latini P. Short-Course Palliative Radiotherapy in Non-Small-Cell Lung Cancer. American Journal of Clinical Oncology: Cancer Clinical Trials. 2000 02;23(1):89-93. https://doi.org/10.1097/00000421-200002000-00024

23. Cross CK, Berman S, Buswell L, Johnson B, Baldini EH. Prospective study of palliative hypofractionated radiotherapy $(8.5 \mathrm{~Gy} \times 2)$ for patients with symptomatic non-smallcell lung cancer. International Journal of Radiation Oncology*Biology*Physics. 2004 03;58(4):1098-1105. https://doi.org/10.1016/j.ijrobp.2003.08.005

\section{(ब) 19}

This work is licensed under a Creative Commons AttributionNon Commercial 4.0 International License. 